\title{
Radio Frequency Tomography for Tunnel Detection
}

\author{
Lorenzo Lo Monte, Student Member, IEEE, Danilo Erricolo, Senior Member, IEEE, \\ Francesco Soldovieri, Michael C. Wicks, Fellow, IEEE
}

\begin{abstract}
Radio Frequency (RF) Tomography is proposed to detect underground voids, such as tunnels or caches, over relatively wide areas of interest.

The RF tomography approach requires a set of low-cost transmitters and receivers deployed randomly on the surface of the ground, or slightly buried. Using the principles of inverse scattering and diffraction tomography, it is possible to develop a simplified theory for below-ground imaging, thus revealing and locating buried objects and hidden targets.

In this work, we introduce the principles and our motivations in support of RF tomography. Furthermore, we derive simple inversion schemes for sensors randomly deployed in a 3D region. Then, we assess limitations to performance, and discuss some system considerations. Finally, we demonstrate the effectiveness of RF Tomography by presenting images reconstructed via the processing of synthetic data.
\end{abstract}

Index Terms-Ground Penetrating Radar, RF Tomography, Inverse Scattering, Tunnel Detection, Buried Object Detection.

\section{INTRODUCTION}

$I^{1}$ $\mathrm{N}$ recent years, the problem of underground void detection and localization has become a critical task, particularly for protecting national borders and for monitoring sensitive areas, such as prisons, banks, and power plants. Additionally, tunnel detection is imperative for civil applications, including mining safety, search and rescue in devastated areas, environmental engineering, geophysics, archaeology and speleology.

Presently, information concerning voids beneath the ground is typically obtained using different techniques, such as microgravity (MG) [1]-[3], electrical resistivity tomography (ERT) [4]-[5], seismic sensing [6]-[8], magnetotellurics (MT) [2], [9], and ground penetrating radars (GPR) [10]-[11].

Manuscript Received December 2008, revised, published. This work was partially supported by the Air Force Research Laboratory, under contract \#F33601-02-F-A581

Lorenzo Lo Monte is with General Dynamics Information Technology, 5100 Springfield Pike, Suite 504, Dayton, OH 45431, USA. Email: lorenzo.lomonte@gdit.com.

Danilo Erricolo is with the University of Illinois at Chicago, $851 \mathrm{~S}$. Morgan Ave, Chicago, IL 60607. Email : erricolo@ece.uic.edu.

Francesco Soldovieri is with the Istituto per il Rilevamento Elettromagnetico dell'Ambiente - CNR, 80124, Naples, Italy. Email: soldovieri.f@irea.cnr.it.

Michael C. Wicks is with the Air Force Research Laboratory, Sensors Directorate, 26 Electronic Parkway, Rome NY 13441. Email: michael.wicks@rl.af.mil.

Digital Object Identifier :
Unfortunately, MG is used for shallow targets, while MT is more appropriate for very deep targets [9], ERT may not work adequately when the impedance of the ground is high, and seismic methods suffer from strong external noise, lack of polarization diversity, weathered soils, and from high attenuation typically encountered [12].

GPR appear to be the most promising and versatile approach. Still, it cannot be considered a reliable and practical method in tunnel detection for the following reasons. First, the resolution for classical GPRs is generally improved by using large bandwidth, often requiring high frequencies. However, due to soil properties, higher frequencies experience higher attenuations, and the increased frequency/bandwidth decreases the signal to noise ratio (SNR), and intensifies dispersion effects. Second, systems using lower frequencies [13] require electrically small wideband antennas, which results in elaborate wideband systems; yet, they may not provide adequate resolution to determine the geometry of targets. Third, the available frequency spectrum for some applications can be severely limited by external sources (e.g. broadcasting stations); therefore, a reliable system must operate using a small, discrete and selectable number of frequencies. Fourth the interpretation of the raw data is affected by the operator's expertise and a priori information is necessary to obtain reliable results [10].

A way to overcome the attenuation losses (thus enabling detection at large investigation depths) has been achieved by emplacing GPR in boreholes [12]-[24], which generally provides an image of a vertical section in the plane of the logs. However, for the important task of tracing tunnel pathways and localizing adits, it is more desirable to perform "horizontal" prospecting, leaving as second priority the determination of their depth. Furthermore, boreholes are expensive, subject to drilling misalignments and, most important, are unpractical in inaccessible terrains.

The improvement of resolution may be accomplished by implementing the principles of RF/Microwave tomography in the framework of GPR [25]-[31], referred to as GPRT. Although an improvement in reconstructed images may be achieved, GPRT still fails to be reliable for tunnel detection for several reasons, including: 1) GPRT is typically employed in a bi-static configuration, where the receive and transmit antennas are separated by an electrically small distance, while operating in proximity to the air-earth interface, therefore it suffers from limited view diversity (using finite observation domains) [32]. 2) To compensate for this limited view, an 
increase in the amount of information is achieved by using multitude of tones, thus making the system wideband [28], [31], [33]. 3) Any GPR require the operator to be above the area under investigation. To date, no system has been designed to work effectively in remote situations where one is disallowed to reach the area of regard.

This article extends, further improves upon, and gives a unified framework to preliminary ideas in RF Tomography proposed in [33], [37]-[42]. In particular, Section II describes the concept of RF Tomography for below-ground imaging, Section III illustrates the principles of RF tomography, presenting the related forward model. Section IV describes several inversion schemes. Finally, Section V shows several reconstructed images obtained via the inversion techniques described in Section IV.

\section{RF TOMOGRAPHY}

To address the unsolved problems in Section I, we introduced a new approach based on a multi-view and multistatic configuration, where the view (associated with the distributed transmitters) and the observation (associated with the multiple receivers) diversities increase the information concerning the scene [34]-[35], while reducing the spectral content of the probing waveform. In fact, in principle, with just a single frequency, it is possible to obtain high resolution images [34], [36].

The proposed approach considers two separate sets of $N$ electromagnetic transmitters and $M$ electromagnetic receivers (commonly referred with the generic name of Transponder). These transponders are placed on the ground at an arbitrary position properly defined by the operator. The Tx radiates a known waveform and exploits the diversity provided by the polarization state. When the probing wave impinges a buried dielectric or conductive anomaly a scattered wavefield is generated. The receivers sample the scattered electric field and estimate the complex valued electric field phasor at their locations. Subsequently, this information is relayed to a data collector. To ease the detection process, one transmitter and frequency of operation per time are activated, thus simplifying the receivers capability to properly discern the origin of the incoming wave-field. For a given sampling time, the used spectrum is restricted to the minimum band achievable, thus ensuing ultra-narrowband system architecture, low noise and affordable cost. To ease the set-up and portability of the system, sensors are intended to be "dart" shaped, as shown in Fig. 2. Sensors may be equipped with built-in GPS for precision timing and positioning, and an opportune S-Band communication link to transfer the collected data to the overhead base station. During the drop-out process, some sensors may end in obstructed regions, or they may fall in region where clutter is excessive (e.g. Tx and Rx lay in proximity, or they may fall over a vegetation layer) and eventually they may default. Indeed, the proposed reconstruction process accounts the eventual failure or obstruction of transponders, by properly neglecting corrupted sensors.

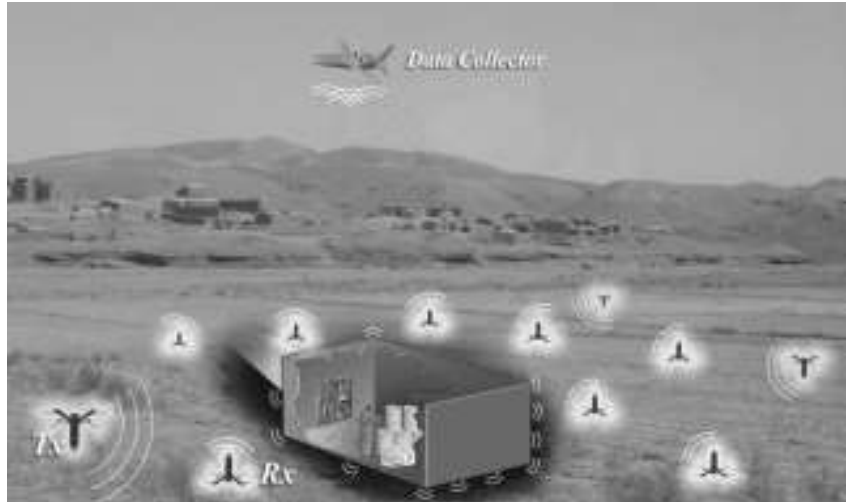

Figure 1: RF Tomography. Transmitters send power into the ground. Receivers collect the scattered field and send this information to the main station.

RF tomography may also operate using a discrete set of monochromatic components, opportunely selected depending on environmental conditions. A suitable modulation is the stepped FM; however, in this paper we derive conclusions that are independent from the type of modulation. The operating frequencies must allow the electromagnetic wave to penetrate deeply into the ground while simultaneously provide acceptable detection and resolution capabilities. Higher frequencies lead to better resolution, but strong attenuation limits the range of operation [60]. Conversely, if the frequencies are low, the corresponding resolution may be not adequate to localize tunnels, and the field behavior becomes diffusive, thus reducing the back-scattered field [29]. Based upon electrical parameters of rocks reported in [10], [61]-[62], a suitable range of frequencies for this application is the range $1-15 \mathrm{MHz}$, but the final choice strictly depends on the expected target type and/or depth.

When antennas are located below-ground, three different modes of propagation between transmitter and scatterer, and between scatterer and receiver, are excited: direct, reflected and lateral waves [52]-[54]. Generally, the lateral mode of propagation is the most undesired: although it could be estimated and removed, its contribution to the overall field may be so high that it may saturate the LNA at the receiver side, thus masking the weak signal coming from the scatterer.

Using vertical dipoles [40] [56], or two vertical ferrite loaded coils [55] as gradiometer antennas, [41], [18], [58], [55] [52], and a proper metallic cover coated with highly lossy materials, we may reduce the effect of lateral waves [42]. However, we remind that the proposed method is valid in principle for any environment, and the reconstruction accuracy depends primarily on the proper choice of the Green's function (see Section III for details). 


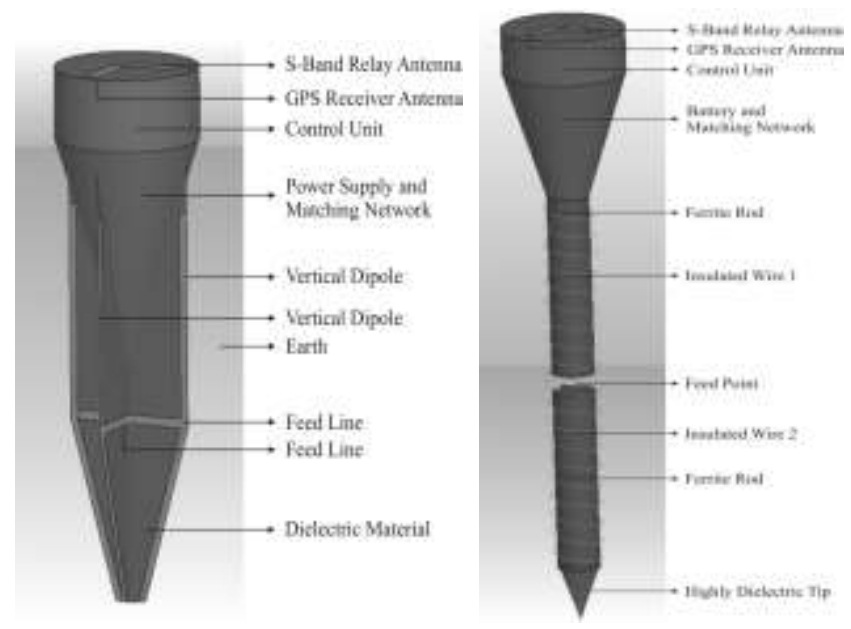

Figure 2: proposed system design for transmitters (left) and receivers (right).

\section{FORWARD MODEL}

The first step to derive a generalized tomographic inversion procedure is to describe a suitable forward model for the electric field that closely represents the actual scene, while keeping enough simplicity to allow for subsequent inversion.

Let us consider the 3D geometry depicted in Fig. 3. For simplicity, a single operating frequency $f$ is adopted, but extension to the multi frequency operation is straightforward [40]. Under the monochromatic assumption, the earth is modeled as a homogeneous medium with constant relative dielectric permittivity $\varepsilon_{D}$, conductivity $\sigma_{D}$, and magnetic permeability $\mu_{0}$. The targets (i.e. tunnels or voids) are assumed to reside in the investigation domain $D$. The sources are $N$ electrically small dipoles (of length $\Delta l^{t}$ ) or loops (of area $A^{t}$ ) fed with current $I^{t}$, and located at position $\mathbf{r}_{n}^{t}$ (view diversity). For each transmitting antenna, the scattered field $\mathbf{E}^{S}$ is collected by $M$ receivers (observation diversity), located at $\mathbf{r}_{m}^{r}$ points in space.

We assume the relative dielectric permittivity profile $\varepsilon_{r}\left(\mathbf{r}^{\prime}\right)$ and the conductivity profile $\sigma\left(\mathbf{r}^{\prime}\right)$ inside the investigation domain $D$ as unknowns of the problem.

Accordingly, the inverse problem is recast in terms of the unknown dielectric permittivity contrast function [35], [43]:

$$
\varepsilon_{\delta}\left(\mathbf{r}^{\prime}\right)=\varepsilon_{r}\left(\mathbf{r}^{\prime}\right)-\varepsilon_{D}+j \frac{\sigma\left(\mathbf{r}^{\prime}\right)-\sigma_{D}}{2 \pi f \varepsilon_{0}}
$$

In this way, the wave number inside $D$ can be expressed as:

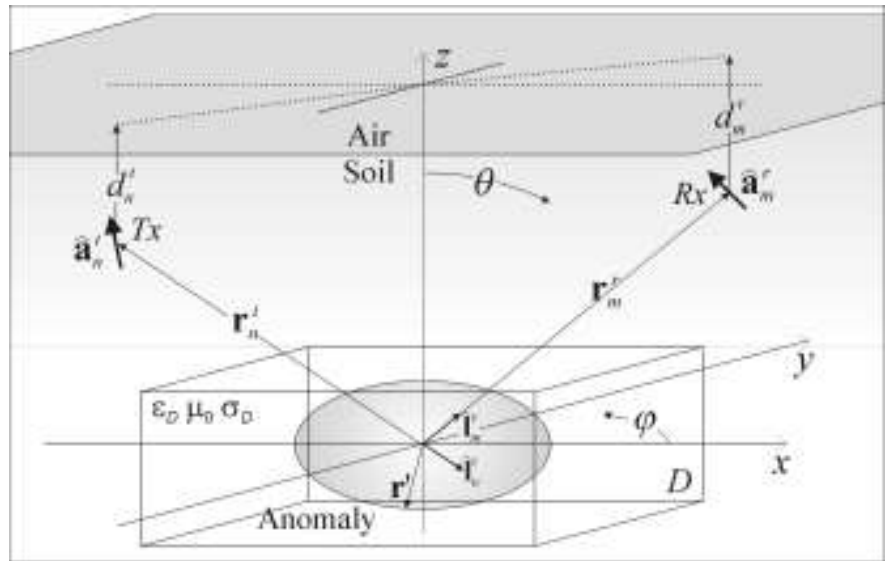

Figure 3: 3D Geometry for the inversion model.

$$
\begin{aligned}
k^{2}\left(\mathbf{r}^{\prime}\right) & =\omega^{2} \mu_{0} \varepsilon_{0} \varepsilon_{r}\left(\mathbf{r}^{\prime}\right)+j \omega \mu_{0} \sigma\left(\mathbf{r}^{\prime}\right), \\
= & k_{D}^{2}+k_{0}^{2} \varepsilon_{\delta}\left(\mathbf{r}^{\prime}\right) \\
k_{D} & =\omega \sqrt{\mu_{0} \varepsilon_{0} \varepsilon_{D}+j \mu_{0} \sigma_{D} / \omega} \\
k_{0} & =\omega \sqrt{\mu_{0} \varepsilon_{0}}
\end{aligned}
$$

The function in (1) accounts for the difference between the unknown dielectric permittivity of the object and that of the host medium. We also accounted for the conductivity profile in (1) and (3), because, in general, the interior surface of a tunnel is humid, steel reinforced or equipped with metal structures that sharply increase the apparent conductivity [12].

For each point $\mathbf{r}^{\prime}$ in region $D$, the vector wave equation holds

$$
\nabla \times \nabla \times \mathbf{E}\left(\mathbf{r}^{\prime}\right)=\left[k_{D}^{2}+k_{0}^{2} \varepsilon_{\delta}\left(\mathbf{r}^{\prime}\right)\right] \mathbf{E}\left(\mathbf{r}^{\prime}\right)
$$

The scattered wave in a point $\mathbf{r} \notin D$ that is solution of (4) can be written in terms of integral equation of the dyadic Green's function

$$
\mathbf{E}^{S}(\mathbf{r})=k_{0}^{2} \iiint_{D} \underline{\underline{G}}\left(\mathbf{r}, \mathbf{r}^{\prime}\right) \cdot \mathbf{E}\left(\mathbf{r}^{\prime}\right) \varepsilon_{\delta}\left(\mathbf{r}^{\prime}\right) d \mathbf{r}^{\prime}
$$

where $\mathbf{E}\left(\mathbf{r}^{\prime}\right)$ is the total field in the investigation domain $D$, given as the superposition of the incident field $E^{I}\left(\mathbf{r}^{\prime}\right)$ (i.e. the field in the investigated area when objects are absent) and the field $\mathbf{E}^{S}(\mathbf{r})$, scattered by the targets.

As it is well known, the inverse scattering problem in (5) is non-linear. Nevertheless, it can be recast as a linear problem by means of the Born approximation (BA). In fact, eq. (5) can be represented in operator form: 


$$
\begin{aligned}
& \mathbf{E}^{S}(\mathbf{r})+\mathbf{E}^{I}(\mathbf{r})=\mathbf{E}(\mathbf{r})=\Gamma \Psi \mathbf{E}(\mathbf{r})+\mathbf{E}^{I}(\mathbf{r}) \\
& \mathbf{E}(\mathbf{r})=\frac{1}{1-\Gamma \Psi} \mathbf{E}^{I}(\mathbf{r})
\end{aligned}
$$

Where $\Gamma$ represents the operation of convolution, and $\Psi$ represents the operation of multiplication by $\varepsilon_{\delta}$. If the operator norm of $\Gamma \Psi$ is less than 1 , then we can expand eq. (6) using Neumann series [57]:

$$
\mathbf{E}(\mathbf{r})=(1+\Gamma \Psi+\Gamma \Psi \Gamma \Psi+\ldots) \mathbf{E}^{I}(\mathbf{r})
$$

The first order Born approximation accounts only for the $(1+\Gamma \Psi)$ term, thus the total field inside the integrand of (5) can be approximated by the known incident field [64], yielding:

$$
\mathbf{E}^{S}(\mathbf{r}) \cong k_{0}^{2} \iiint_{D} \underline{\underline{\mathbf{G}}}\left(\mathbf{r}, \mathbf{r}^{\prime}\right) \cdot \mathbf{E}^{I}\left(\mathbf{r}^{\prime}\right) \varepsilon_{\delta}\left(\mathbf{r}^{\prime}\right) d \mathbf{r}^{\prime}
$$

Therefore, the inverse problem at hand is cast as the inversion of the linear integral equation connecting the permittivity contrast function to the scattered field data. The use of BA can be justified by considering that:

- Tunnels (or other targets of interest) are isolated, limited in number and embedded in a lossy medium. Therefore, mutual interaction, a phenomenon ignored by BA, between tunnels can be assumed negligible.

- In general, the inhomogeneities of the soil are electrically small, and their conductivity remains low. Therefore, their scattered fields are insignificant compared to the RF signal re-irradiated by tunnels.

- The operator norm of $\Gamma \Psi$ is generally $\square 1$, due to the exponential loss factor of the medium [68], and to the spherical spreading factor of the incident and scattered wave.

- Our goal is to detect, localize, and approximately determine the geometry of the targets. Toward this objective, BA based inversion algorithms preserve the information on target localization, even when objects are strong scatterers (although the quantitative description of $\varepsilon_{\delta}$ in $D$ is generally altered) [30], [43].

The incident field, i.e. the field radiated in the earth medium from a point source located at position $\mathbf{r}_{n}^{t}$ can be expressed in terms of Green's functions as

$$
\mathbf{E}_{n}^{I}\left(\mathbf{r}^{\prime}, \mathbf{r}_{n}^{t}\right)=Q \underline{\underline{G}}\left(\mathbf{r}^{\prime}, \mathbf{r}_{n}^{t}\right) \cdot \hat{\mathbf{a}}_{n}^{t},
$$

where $Q=j \omega \mu_{0} \Delta l^{t} I^{t}$ for an electrically small dipole, or $Q=-j \omega \mu_{0} A^{t} I^{t}$ for an electrically small loop and $\hat{\mathbf{a}}_{n}^{t}$ is the (electric or magnetic) dipole moment direction.

Additionally, the field received by a dipole or loop with moment direction $\hat{\mathbf{a}}_{m}^{r}$ positioned at $\mathbf{r}_{m}^{r}$ due to an equivalent (in terms of $\mathbf{E}_{n}^{I}$ ) current distribution defined inside the investigation domain $D$ can be expressed as [28]

$$
\begin{aligned}
& E^{S}\left(\mathbf{r}_{n}^{t}, \mathbf{r}_{m}^{r}\right)=k_{0}^{2} \times \\
& \iiint_{D} \hat{\mathbf{a}}_{m}^{r} \cdot \underline{\underline{\mathbf{G}}}\left(\mathbf{r}_{m}^{r}, \mathbf{r}^{\prime}\right) \cdot \mathbf{E}_{n}^{I}\left(\mathbf{r}^{\prime}, \mathbf{r}_{n}^{t}\right) \varepsilon_{\delta}\left(\mathbf{r}^{\prime}\right) d \mathbf{r}^{\prime}
\end{aligned}
$$

Substituting (9) in (10) we obtain the scalar forward model for the scattered field is

$$
\begin{aligned}
& E^{S}\left(\mathbf{r}_{n}^{t}, \mathbf{r}_{m}^{r}\right)=\mathbf{L}\left(\varepsilon_{\delta}\left(\mathbf{r}^{\prime}\right)\right)=Q k_{0}^{2} \times \\
& \iiint_{D}\left[\mathbf{a}_{m}^{r} \cdot \underline{\underline{\mathbf{G}}}\left(\mathbf{r}_{m}^{r}, \mathbf{r}^{\prime}\right)\right] \cdot\left[\underline{\underline{\mathbf{G}}}\left(\mathbf{r}^{\prime}, \mathbf{r}_{n}^{t}\right) \cdot \mathbf{a}_{n}^{t}\right] \varepsilon_{\delta}\left(\mathbf{r}^{\prime}\right) d \mathbf{r}^{\prime}
\end{aligned}
$$

From (11) and (9), we can also determine the total field experienced at the receiver in time domain

$$
\begin{aligned}
& E\left(\mathbf{r}_{n}^{t}, \mathbf{r}_{m}^{r}, t\right)=e^{-j \omega t} Q k_{0}^{2} \times \\
& \iiint_{D}\left[\mathbf{a}_{m}^{r} \cdot \underline{\underline{\mathbf{G}}}\left(\mathbf{r}_{m}^{r}, \mathbf{r}^{\prime}\right)\right] \cdot\left[\underline{\underline{\mathbf{G}}}\left(\mathbf{r}^{\prime}, \mathbf{r}_{n}^{t}\right) \cdot \mathbf{a}_{n}^{t}\right] \varepsilon_{\delta}\left(\mathbf{r}^{\prime}\right) d \mathbf{r}^{\prime}( \\
& +e^{-j \omega t} Q \mathbf{a}_{m}^{r} \cdot \underline{\underline{\mathbf{G}}}\left(\mathbf{r}_{m}^{r}, \mathbf{r}_{n}^{t}\right) \cdot \mathbf{a}_{n}^{t}+N(t)
\end{aligned}
$$

The second term represents the direct coupling between Tx and $\mathrm{Rx}$, and it can be considerer a source of deterministic clutter. The random variable $N(t)$ can be modeled as Gaussian process with zero mean and variance equal to the noise power

$$
P_{N}=F_{a}+10 \log B+10 \log \left[K_{B} T_{0}\right] \quad[\mathrm{dBW}](13)
$$

Where $B$ is the bandwidth, $K_{B}$ is the Boltzmann constant, $T_{0}$ is the environmental temperature, and the external noise figure $F_{a}$ can be inferred by consulting [63].

Tomography is inherently suited for noise mitigation, since it is ultra-narrowband (therefore, $P_{N}$ is intrinsically very low), and by simply averaging $n$ samples of the same signal we obtain a theoretical SNR increase of $\sqrt{n}$. Furthermore, the clutter contribution in (12) is generally low-correlated with the value of the scattered field, meaning that the combination of view and observation diversities randomizes the static clutter. This implies that, by sampling the field at different locations, 
the static clutter may be treated as a compound noise with zero mean, and, similarly to the time domain case, the overall SNR increases by a factor (at least in principle) proportional to $\sqrt{N M}$.

To obtain the electric field in phasor form from (12), the instantaneous received field can be mixed with two coherent oscillators to retrieve the in-phase and quadrature components. In fact, the real and imaginary phasor components are:

$$
\begin{aligned}
& \mathbf{E}\left(\mathbf{r}_{n}^{t}, \mathbf{r}_{m}^{r}, t\right)=\operatorname{Re}\left[\mathbf{E}\left(\mathbf{r}_{n}^{t}, \mathbf{r}_{m}^{r}\right) \exp (-j \omega t)\right]= \\
& \operatorname{Re}\left[\mathbf{E}\left(\mathbf{r}_{n}^{t}, \mathbf{r}_{m}^{r}\right) \cos (\omega t)\right]+\operatorname{Im}\left[\mathbf{E}\left(\mathbf{r}_{n}^{t}, \mathbf{r}_{m}^{r}\right) \sin (\omega t)\right]
\end{aligned}
$$

Hence, the real and imaginary part can be inferred by integration over the period $T$ :

$$
\begin{aligned}
& \operatorname{Re}\left[\mathbf{E}\left(\mathbf{r}_{n}^{t}, \mathbf{r}_{m}^{r}\right)\right]=\frac{2}{T} \int_{t}^{t+T} \mathbf{E}\left(\mathbf{r}_{n}^{t}, \mathbf{r}_{m}^{r}, t\right) \cos (\omega t) d t \\
& \operatorname{Im}\left[\mathbf{E}\left(\mathbf{r}_{n}^{t}, \mathbf{r}_{m}^{r}\right)\right]=\frac{2}{T} \int_{t}^{t+T} \mathbf{E}\left(\mathbf{r}_{n}^{t}, \mathbf{r}_{m}^{r}, t\right) \sin (\omega t) d t
\end{aligned}
$$

\section{INVERSION PROCEDURES}

From a mathematical point of view, the problem of finding the dielectric contrast function is to compute the inverse of the linear operator $\mathbf{L}$ in (11). We are also assuming that noise and clutter contributions are minimal so that they can be considered as perturbations on the measured data.

A way to compute $\mathbf{L}^{-1}$ is to perform a numerical inversion of $\mathbf{L}$. Let us collect the sampled field data in an ordered vector $\underline{E}^{S}=\left\{E^{S}\left(\mathbf{r}_{n}^{t}, \mathbf{r}_{m}^{r}\right)\right\}$ of length $N M$, and discretize the domain region $D$ in $K$ voxels, each one located at position $\mathbf{r}_{k}{ }^{\prime}$ : the contrast dielectric permittivity can be embodied in a column vector $\underline{\varepsilon}_{\delta}=\left\{\varepsilon_{\delta}\left(\mathbf{r}_{k}{ }^{\prime}\right)\right\}$ of length $K$, and it represents the set of unknown parameters. After this discretization, eq. (11) can be rewritten in a matrix form

$$
\underline{E}^{S}=\underline{\underline{\mathbf{L}}} \underline{\varepsilon}_{\delta}
$$

where $\mathbf{L}$ now is a matrix with dimensions $N M \times K$, and $\underline{E}^{S}, \underline{\varepsilon}_{\delta}$ are column vectors.

The problem is then to invert the relation (17). Since $\mathbf{L}$ is not a square matrix, we need to consider its pseudoinverse that we still indicate with $L^{-1}$. Due to the usual location of Tx, Rx and targets, $\mathbf{L}$ is generally severely ill conditioned. A common way to quantify the behavior of $\mathbf{L}$ is by inspection of its condition number $\kappa$. For the operator $\mathbf{L}$ it is quite common to obtain values of $\kappa$ above $10^{6}$. This leads to artifacts in the reconstruction process, particularly exacerbated when noise (thermal, external, quantization) or clutter is impinging on the receivers.

According to the accuracy required from the system, we present four inversion strategies:

- Levenberg-Marquardt (LM) regularization procedure. This method is relatively accurate for any environmental condition and it is robust in presence of noise. It requires a proper choice of a regularization parameter.

- Truncated Singular Value Decomposition (TSVD). This method is also relatively accurate in any scenario and fairly resistant to noise interference. This method also offers deeper insight into the physics behind the reconstruction, and the output can be easily adjusted by properly selecting the number of meaningful singular values. Also, the number of retained singular values in TSVD plays the same role of the LM regularization parameter.

- Back Propagation approach. This method works properly only when the operator $\mathbf{L}$ is well conditioned. This implies that it can be used only for particular configurations and when the SNR is relatively high. However, the computational time is drastically reduced.

- Fourier-Bojarski approach. This is the fastest inversion scheme and it is suited for far field probing and nearlossless Green's function. It privileges speed instead of accuracy.

\section{A. Levenberg-Marquardt Regularization}

An efficient method to compute the inverse of an ill conditioned matrix is by using the Levenberg-Marquardt regularization procedure [2]. In this way, the contrast dielectric permittivity is estimated as

$$
\underline{\hat{\varepsilon}}_{\delta}=\left(\underline{\underline{\mathbf{L}}}^{H} \underline{\underline{\mathbf{L}}}+\beta \underline{\underline{\mathbf{I}}}\right)^{-1} \underline{\underline{\mathbf{L}}}^{H} \underline{E}^{S},
$$

where $\mathbf{L}^{H}$ denotes the adjoint of $\mathbf{L}$, and $\beta$ is the regularization parameter in the Tikhonov sense, which needs to be advantageously selected. Since a proper choice of $\beta$ may be a difficult task, our initial guess for $\beta$ is the mid-point value of the singular value dynamic range of $\mathbf{L}$. Sometimes, it is necessary to determine $\beta$ through a constrained optimization solution before a meaningful, sharp and low blurred image is reconstructed [2], [69]. This implies a (computationally expensive) matrix inversion for each attempt that may become necessary. To accelerate this process, the SVD decomposition may be used, which is described next.

\section{B. Singular Value Decomposition}

A more efficient way to invert the ill conditioned matrix $\mathbf{L}$ was proposed in [34]-[35], [43], and takes advantage of 
singular value decomposition. In fact, $\mathbf{L}$ can be decomposed as

$$
\underline{\underline{\mathbf{L}}}=\underline{\underline{\mathbf{U}}} \underline{\underline{\mathbf{S}}} \underline{\underline{V}}^{H}
$$

where $\mathbf{S}$ is a diagonal matrix containing the ordered singular values $s_{i}$ of $\mathbf{L}$. The pseudoinverse of $\mathbf{L}$ can be written as:

$$
\underline{\underline{\mathbf{L}}}^{\dagger}=\underline{\underline{\mathbf{V}}}_{\underline{\underline{\mathbf{U}}}}^{\dagger} \underline{\underline{\mathbf{U}}}^{H} \text { (FIX THIS EQUATION) }
$$

Singular values of $S^{-1}$ that are considerably large compared to $1 / s_{1}$ represent the sensitive directions of $\mathbf{L}$ : along these directions, small amounts of noise or clutter in the sampled electric field will lead to a large (undesired) deviation of $\varepsilon_{\delta}$.

A way to remove this sensitivity is to consider only the first $k$ smaller singular values of $S^{-1}$ and setting to zero the remaining large ones. This strategy is commonly referred to as "Truncated SVD" [66]. In this way, the dielectric profile can be estimated as

$$
\hat{\boldsymbol{\varepsilon}}_{\delta}=\mathbf{V} \mathbf{S}_{k}^{\dagger} \mathbf{U}^{H} .(\text { FIX THIS FORMULA) }
$$

The SVD method can be also very useful to properly dimension the $\beta$ parameter in the LM method. In fact, if we rewrite (18) in terms of (19) we obtain:

$$
\begin{aligned}
\underline{\underline{\mathbf{L}}}^{\dagger} & =\left(\underline{\underline{\mathbf{L}}}^{H} \underline{\underline{\mathbf{L}}}+\beta \mathbf{I}\right)^{-1} \underline{\underline{\mathbf{L}}}^{H} \\
& =\mathbf{V} \operatorname{diag}\left(\frac{s_{i}^{2}}{s_{i}^{2}+\beta} \frac{1}{s_{i}}\right) \mathbf{U}^{H}
\end{aligned}
$$

(THE EQUATION SHOULD FIT IN ONE LINE)

Truncated SVD and the SVD representation of the LM method in (22) have a remarkable feature. In both cases, once the evaluation of the singular system of $\mathbf{L}$ is performed, (19), the reconstructed image is simply computed by a (fast) matrix multiplication, as in (20). This means that a new image is obtained by varying the number of singular values (for the truncated SVD method) or varying $\beta$ (for the LM method), and it can be computed extremely fast (often in real time).

\section{Back Propagation}

The approximate number of computations to perform the SVD of $\underline{\underline{\mathbf{L}}}$ is $9 K^{3}+12 M N K^{2}$ [47]. When the size of the matrix is large, the evaluation of (19) may become prohibitive and an alternative efficient strategy has to be pursued. In this case, we choose to invert equation (17) by estimating the contrast permittivity function as

$$
\hat{\boldsymbol{\varepsilon}}_{\delta}=\left(\underline{\mathbf{L}}^{H} \underline{\underline{\mathbf{L}}}\right)^{-1} \underline{\mathbf{L}}^{H} \underline{E}^{S} \cong \underline{\mathbf{L}}^{H} \underline{E}^{S}
$$

where the computation is performed usin the adjoint matrix. The inversion by means of the adjoint matrix holds theoretically when:

$$
\underline{\underline{\mathbf{L}}} \underline{\underline{\mathbf{L}}}^{H}=\underline{\underline{\mathbf{L}}}^{H} \underline{\underline{\mathbf{L}}}=\alpha \underline{\underline{\mathbf{I}}} \quad \alpha \in \mathfrak{R},
$$

where $\alpha \underline{\underline{\mathbf{I}}}$ represents a scaled identity matrix. This result implies that, in principle, $\kappa\left(\underline{\underline{\mathbf{L}}}^{H} \underline{\underline{\mathbf{L}}}\right)=1$. However [47]

$$
\kappa\left(\underline{\underline{\mathbf{L}}}^{H} \underline{\underline{\mathbf{L}}}\right)=\kappa\left(\underline{\underline{\mathbf{L}}}^{H}\right)^{2}=\kappa(\underline{\underline{\mathbf{L}}})^{2}
$$

Therefore, the use of the adjoint for the inversion can be considered a good tool only when the singular values of $\underline{\underline{\mathbf{L}}}$ exhibit a limited dynamic range. In this case, it is possible to give an explicit formula for the solution of (23) in terms of each $\mathbf{r}_{k}{ }^{\prime}$ :

$$
\begin{aligned}
& \hat{\varepsilon}_{\delta}\left(\mathbf{r}_{k}^{\prime}\right)= Q^{*} k_{0}^{2} \sum_{n=1}^{N} \sum_{m=1}^{M}\left(\mathbf{a}_{m}^{r} \cdot \underline{\underline{G}}^{*}\left(\mathbf{r}_{m}^{r}, \mathbf{r}_{k}^{\prime}\right)\right) . \\
&\left(\underline{\underline{G}}^{*}\left(\mathbf{r}_{n}^{t}, \mathbf{r}_{k}^{\prime}\right) \cdot \mathbf{a}_{n}^{t}\right) E^{S}\left(\mathbf{r}_{n}^{t}, \mathbf{r}_{m}^{r}\right)
\end{aligned}
$$

Eq. (26) is commonly referred to as matched filtering, migration or back-propagation [67]. This technique is suited for parallel processing [33].

\section{Fourier-Bojarski Approach}

A simple and fast approach (albeit less accurate) is to take advantage of the Fourier relation arising between scattered field and object shape, as discussed in the literature about diffraction tomography.

In fact, if targets and sensors are distant enough so that the propagating wave is TEM (normally occurring when the fields are primarily propagating as $1 / r$ ), then the forward model can be expressed as explained below.

We define the unit norm direction of propagation vectors as:

$$
\begin{gathered}
\hat{\mathbf{l}}_{n}^{t}=-\hat{\mathbf{x}} \sin \theta_{n}^{t} \cos \varphi_{n}^{t}-\hat{\mathbf{y}} \sin \theta_{n}^{t} \sin \varphi_{n}^{t}-\hat{\mathbf{z}} \cos \theta_{n}^{t}, \\
\hat{\mathbf{l}}_{m}^{r}=\hat{\mathbf{x}} \sin \theta_{m}^{r} \cos \varphi_{m}^{r}+\hat{\mathbf{y}} \sin \theta_{m}^{r} \sin \varphi_{m}^{r}+\hat{\mathbf{z}} \cos \theta_{m}^{r}
\end{gathered}
$$

Using the paraxial approximation, the transmitting Green's function at the generic position $\mathbf{r}^{\prime}$ inside region $D$ can be simplified as:

$$
G_{n}^{t}\left(\mathbf{r}_{n}^{t}, \mathbf{r}^{\prime}\right) \cong \frac{\exp \left(+j k_{D} r_{n}^{t}\right) \exp \left(+j k_{D} \hat{\mathbf{l}}_{n}^{t} \cdot \mathbf{r}^{\prime}\right)}{4 \pi r_{n}^{t}}
$$




$$
G_{m}^{r}\left(\mathbf{r}^{\prime}, \mathbf{r}_{m}^{r}\right) \cong \frac{\exp \left(+j k_{D} r_{m}^{r}\right) \exp \left(-j k_{D} \hat{\mathbf{l}}_{m}^{r} \cdot \mathbf{r}^{\prime}\right)}{4 \pi r_{m}^{r}}
$$

Therefore, for a pair of transmitters and receivers, the scattered field can be rewritten as [36]

$$
\begin{aligned}
E^{S}\left(\mathbf{r}_{n}^{t}, \mathbf{r}_{m}^{r}\right) \cong & \frac{k_{0}^{2} \hat{\mathbf{a}}_{n}^{t} \cdot \hat{\mathbf{a}}_{m}^{r}}{16 \pi^{2} r_{n}^{t} r_{m}^{r}} Q e^{+j k_{D}\left(r_{r}^{t}+r_{m}^{r}\right)} \times \\
& \iiint_{D} \varepsilon_{\delta}\left(\mathbf{r}^{\prime}\right) \exp \left[+j k_{D}\left(\hat{\mathbf{l}}_{n}^{t}-\hat{\mathbf{l}}_{m}^{r}\right) \cdot \mathbf{r}^{\prime}\right] d \mathbf{r}^{\prime},
\end{aligned}
$$

where the quantity $k_{D}\left(\hat{\mathbf{l}}_{n}^{t}-\hat{\mathbf{l}}_{m}^{r}\right)$ can be represented by a 3D vector

$$
\mathbf{k}_{m n}=k_{D}\left(\hat{\mathbf{I}}_{n}^{t}-\hat{\mathbf{I}}_{m}^{r}\right)
$$

Eq. (31) is then rewritten as [33]

$$
\begin{aligned}
E^{S}\left(\mathbf{k}_{m n}\right)= & \frac{k_{0}^{2} \hat{\mathbf{a}}_{n}^{t} \cdot \hat{\mathbf{a}}_{m}^{r}}{16 \pi^{2} r_{n}^{t} r_{m}^{r}} Q e^{+j k_{D}\left(r_{m}^{t}+r_{n}^{r}\right)} \times \\
& \iiint_{D} \varepsilon_{\delta}\left(\mathbf{r}^{\prime}\right) \exp \left(+j \mathbf{k}_{m n} \cdot \mathbf{r}^{\prime}\right) d \mathbf{r}^{\prime} \cdot
\end{aligned}
$$

It is useful to consider a normalized version of the previousequation

$$
\begin{aligned}
\bar{E}^{S}\left(\mathbf{k}_{m n}\right) & =\frac{16 \pi^{2} r_{n}^{t} r_{m}^{r}}{Q k_{0}^{2} \hat{\mathbf{a}}_{n}^{t} \cdot \hat{\mathbf{a}}_{m}^{r}} e^{-j k_{D}\left(r_{n}^{t}+r_{m}^{r}\right)} E^{S}\left(\mathbf{k}_{m n}\right) \\
& =\iiint_{D} \varepsilon_{\delta}\left(\mathbf{r}^{\prime}\right) \exp \left(+j \mathbf{k}_{m n} \cdot \mathbf{r}^{\prime}\right) d \mathbf{r}^{\prime}
\end{aligned}
$$

This result can be interpreted in the following way: each collected sample $\bar{E}^{S}\left(\mathbf{k}_{m n}\right)$ returns the value of the $\mathbf{k}_{m n}$ spectral component of the contrast function $\varepsilon_{\delta}\left(\mathbf{r}^{\prime}\right)$. Theoretically, if we have enough samples to fully populate the spectral representation of $\varepsilon_{\delta}\left(\mathbf{r}^{\prime}\right)$, the discrete function $\bar{E}^{S}\left(\mathbf{k}_{m n}\right)$ can be approximated, in the limit, as a continuous function $\bar{E}^{S}(\mathbf{k})$, and (34) can be interpreted as a 3D inverse Fourier transform of the permittivity contrast function. Therefore, we can reconstruct an image of the underground by direct Fourier transformation of eq. (33), i.e.

$$
\hat{\varepsilon}_{\delta}\left(\mathbf{r}^{\prime}\right)=\iiint_{K} \bar{E}^{S}(\mathbf{k}) \exp \left(-j \mathbf{k} \cdot \mathbf{r}^{\prime}\right) d \mathbf{k}
$$

where the domain of integration $K$ is the support of $\bar{E}^{S}(\mathbf{k})$.
By inspection of (32), we conclude that when the sensors completely encircle the target, $K$ is a sphere of radius $2 k_{D}$. In other words, the available information from the spectral content of $\varepsilon_{\delta}\left(\mathbf{r}^{\prime}\right)$ is limited up to the spectral component contained inside a sphere of radius $2 k_{D}$. Therefore, the reconstructed image of the dielectric profile will be a low pass filtered version of the true image.

By studying the impulse response of (34), we showed that the minimum resolution achievable using Fourier-Bojarski approach is $d \cong \lambda_{D} / 3$ [39]. For half space problems, the resolution is further reduced [49].

In real cases, where a finite number of sensors are deployed (i.e. the spectral domain is undersampled) and external noise affects the measurements, the resolution is lower, and artifacts in the reconstructed image are very common. Severe smearing and blurring effects originate mainly from the invalidity of the paraxial approximation. In fact, paraxial approximation holds when the maximum angle between the ray passing through the origin and the ray intersecting the boundary of the region $D$ is negligible. A way to overcome this limitation is to segment the region $D$ in smaller analysis regions and consider an inverse problem for each sub-region. Then, for each sub-region a (smaller) FFT is carried out, and the resulting sub-images are concatenated to form the final image [39].

The undersampling of the spectral domain can be corrected with several approaches, such as tri-linear interpolation [50] of the available samples, or using projection on convex sets [51] and smoothing of the peaks in the spectral domain to estimate the missing samples [39].

\section{Simulations}

We present some simulation results to test the proposed underground imaging system. A set of 6 transmitters and 26 receivers, operating at $5 \mathrm{MHz}$, are arbitrarily emplaced below ground at a depth $d=0.15 \mathrm{~m}\left(\mathbf{a}^{t}=\mathbf{a}^{r}=\hat{\mathbf{x}}\right)$, as shown in Fig 4. Two empty cylindrical structures of radius $\rho=1 \mathrm{~m}$ (representing two tunnels) are assumed to be embedded in a host medium with relative dielectric permittivity $\varepsilon_{D}=10$, and conductivity $\sigma_{D}=5 \times 10^{-4} \mathrm{~S} / \mathrm{m} \quad$ [62], at a depth $h=25 \mathrm{~m}$. The corresponding attenuation can be computed using [65]

$$
a \cong 8.68 \frac{\sigma_{D}}{2} \sqrt{\frac{\mu_{0}}{\varepsilon_{0} \varepsilon_{D}}} \mathrm{~dB} / \mathrm{m}
$$

The forward scattered field was synthesized using two methods: Born and FDTD. To mimic the real environment, we used the FDTD simulator GPRMAX [70]: the instantaneous scattered electric field has been correlated using (15) and (16) to retrieve the phasor form of the electric field. To show the 
accuracy of the proposed forward model, the scattered electric field has been computed simultaneously using the forward model in (11), and using the Green's dyad for the homogeneous space (with the same properties of the soil), i.e. [44]:

$$
\underline{\underline{\mathbf{G}}}\left(\mathbf{r}, \mathbf{r}^{\prime}\right)=\left[\underline{\underline{\mathbf{I}}}+\frac{1}{k_{D}^{2}} \nabla \nabla \cdot\right] \frac{e^{j k_{D}\left|\mathbf{r}-\mathbf{r}^{\prime}\right|}}{4 \pi\left|\mathbf{r}-\mathbf{r}^{\prime}\right|}
$$

This assumption is reasonable when the sensors are deployed at the air/ground interface, the frequencies involved are relatively low, and targets are assumed to reside nearly perpendicular to sensors. The shallower the scatterer, the higher the lateral mode; furthermore, lateral modes predominate when targets reside at low grazing angles from $\mathrm{Tx}$ or Rx.

Nonetheless, more accurate models can take into account the distortions due to half-space or layered geometry by simply selecting the proper Green's function under a spectral form [28], [44]-[46], or by numerically computing the Green's function using method of moments or fast eikonal equation solvers.

Clutter mitigation was possible because of the exact knowledge of the dyadic Green's function of the problem, which enabled the accurate determination (and subsequent cancellation) of its value. Random Gaussian noise has been added to the data according to [63]. Nevertheless, the SNR can be reduced to a desired value by appropriately sampling and averaging the received field, as discussed in Section IV.

After having performed the 3D tomographic inversion (using a mesh of $1 \mathrm{~m}^{3}$ per voxel), we plotted only the horizontal section at $25 \mathrm{~m}$ depth (constant depth slice), with the aim of better visualizing the performance of the proposed inversion schemes. For more complex scenarios, a full 3D image might be necessary.

All images have been filtered by a hard cancellation for any voxel whose value is less than $5 \%$ of the maximum value.

The inversion procedure using Fourier-Bojarski method has a different (coarser) voxel size equal to $\lambda_{D} / 4$, because sampling at finer discretization does not provide more information (due to the proximity to the resolution limit [39]), while it dramatically increase the sparsity of the Fourier domain, leading to severe artifacts.

Figure 5 shows the validity of the Green's function of the homogeneous space (37): when the error between the theoretical Green's function and the actual electric field passes the value of $100 \%$, measures may become inaccurate. For this reason, we chose to place transmitters and receivers in proximity to the targets, so that the expected error by using the homogeneous Green's function is minimized. It is important to mention that the proximity of sensors is not advantageous for the quality of the reconstructed image: if we could separate sensors, geometric diversity would be increased, but a different Green's function needs to be used.
In Figure 6, a comparison between the scattered field generated by the FDTD simulator, and the scattered field obtained using the forward model presented in Section III is shown. The abscissa represents the sequence of transmitter/receiver pairs, and it is not related to the range. The absolute value is generally confined within a range of $5 \mathrm{~dB}$ : sharp peaks are occurring because the Born field predicts the field to be close to zero, while the mesh generated in the FDTD simulator returns an averaged value within the cell, which is generally not exactly zero. The phase difference between Born field and FDTD generated field is contained within the range of $30^{\circ}$ of error: sharp peaks corresponds to the regions where the field is close to zero, and they are not influencing the overall reconstruction process.

Fig. 7 shows the reconstructed image using the (fast) Fourier-Bojarski method: although the resolution is coarse, basic traces of the two tunnels can be discerned. However, the back-propagation method (see Fig. 8) is clearly showing higher resolution capabilities, while keeping the computational cost at minimum. For high level image reconstruction, regularized methods are paramount. In Figure 9, the image has been reconstructed using LM method (in its SVD variant), and the regularization parameter has been empirically selected in order to achieve the sharpest solution. In Figure 11, an image reconstructed using TSVD is shown: we assume that $10 \%$ of the total singular values represent the sensitive directions of $\mathbf{L}$ and therefore they will be not included in the reconstruction based on TSVD (see Fig. 10). This threshold has been chosen heuristically, and it may vary according to the geometry and the SNR.

\section{CONCLUSIONS}

We proposed a practical method for tunnel detection that does not require boreholes, and is easy to be deployed and that covers relatively wide areas.

We applied diffraction tomography and inverse scattering principles to our geometry. We proposed four simple methods to reconstruct images that are suited for the environment encountered in practical situations.

Finally, we showed several reconstructed images, and in all cases the location of the two tunnels is discernible when noise is low, while tunnel detection amid high noise is only possible when LM and TSVD methods are used.

In particular, by comparing Figs. 7, 8 with Figs. 9, 11, it is clear that LM and TSVD methods yield higher quality images. Therefore, if the computational load is not a consideration, these two methods should be preferred, and become imperative when environmental conditions are hostile.

The proposed strategy offers the following advantages:

- RF tomography is able to surveil from local/shallow to global/deep areas of regard, and rapidly focus on specified areas, by simply changing frequency of operation and the delimitation of the investigation domain $D$.

- The system is suited for both cooperative and denied scenes, where the physical presence of the human operator is hazardous. 
- The ultra-narrowband sensor design and the system architecture are fiscal and manpower affordable solutions.

- The sensor deployment is arbitrary, and modular (i.e. the addition or removal of sensors does not compromise the remainder of the system)

- The resolution of the system is sub-wavelength and rangeindependent.

However, many aspects still need deeper investigations, such as more accurate inversion models, the use of different Green's functions, improved methods of direct path cancellation, or more considerations on the actual soil and antennas behavior.

In particular, the issue of unwanted lateral waves should be addressed in two ways: by investigating clutter suppression techniques, or by defining a suitable Green's function that accounts for this effect. We are currently pursuing further research in both directions.

\section{ACKNOWLEDGEMENTS}

The authors are thankful to Mr. W. Baldygo, Air Force Research Laboratory, and Dr. J.A. Sjogren, Air force Office of Scientific Research, for sponsoring and funding this research. We are also grateful to Mr. J. Parker and Dr. M. Ferrara, Air Force Research Laboratory, Prof. R. Ansari, University of Illinois at Chicago, and Prof. M. Cheney, Rensselaer Polytechnic Institute, for their technical discussions.

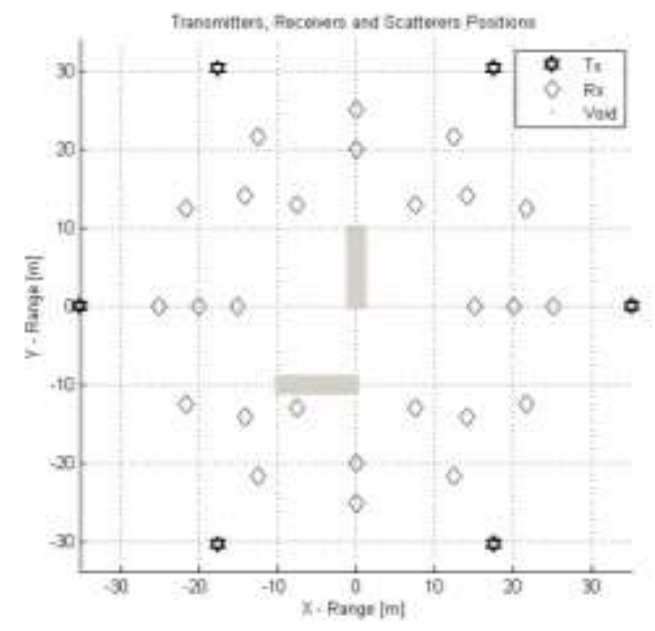

Figure 4: Geometry for the simulation (top view).

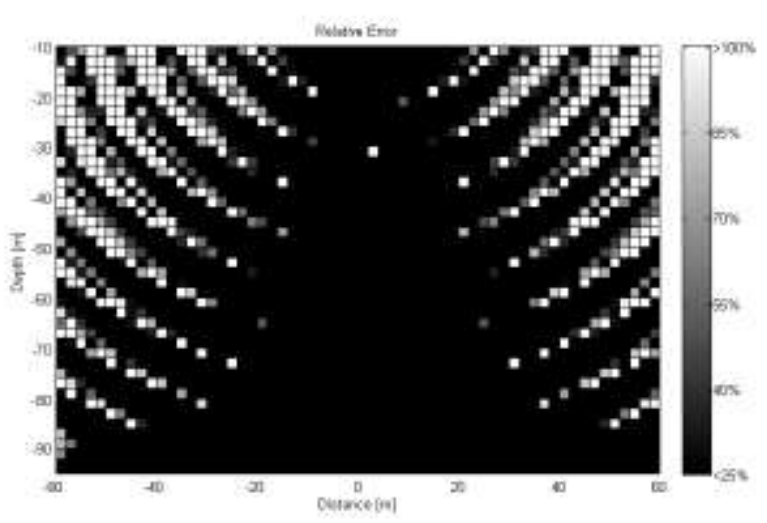

Figure 5: snapshot of the relative error between the transmitted field of a source in homogeneous medium, and a source in an half-space medium, as described in Section V.(HOW DO YOU DEFINE THE RELATIVE ERROR?)
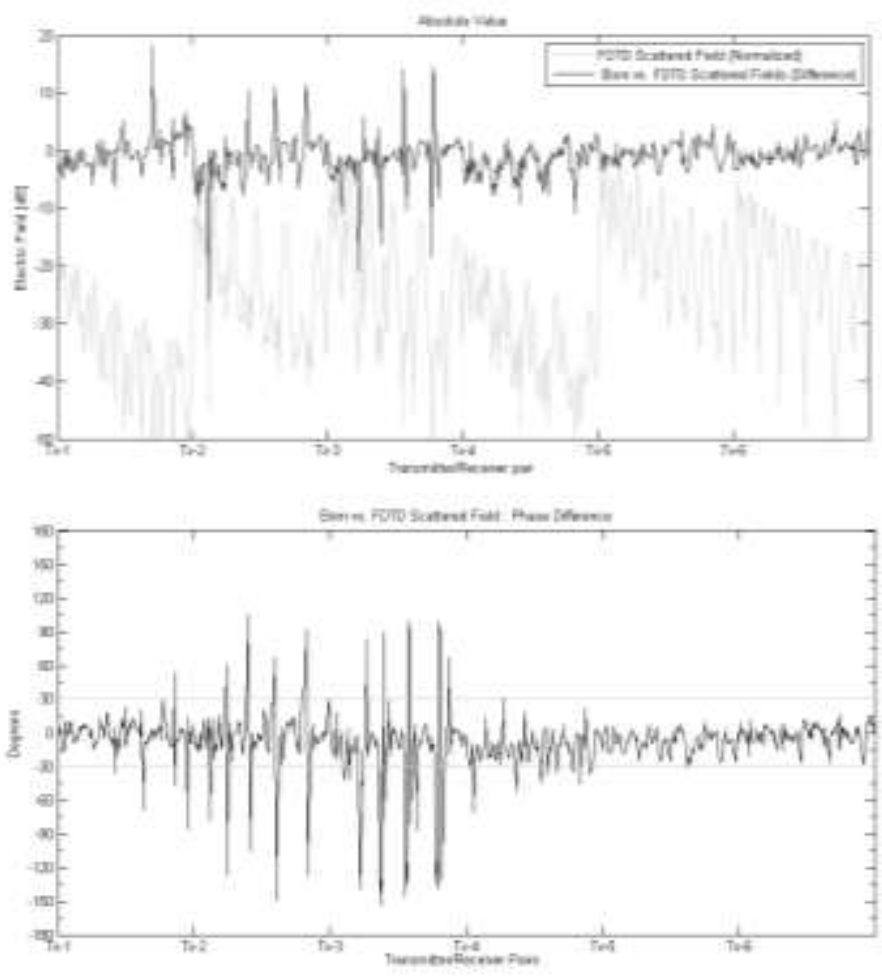

Figure 6: comparison between scattered field generated by FDTD simulation, and scattered field obtained using Born approximation: amplitude difference (top) and phase difference (bottom). 


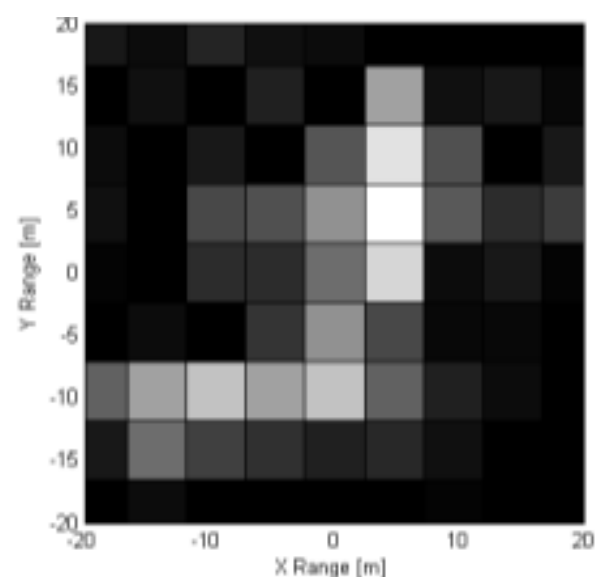

Figure 7: Reconstructed image using Fourier-Bojarski approach.

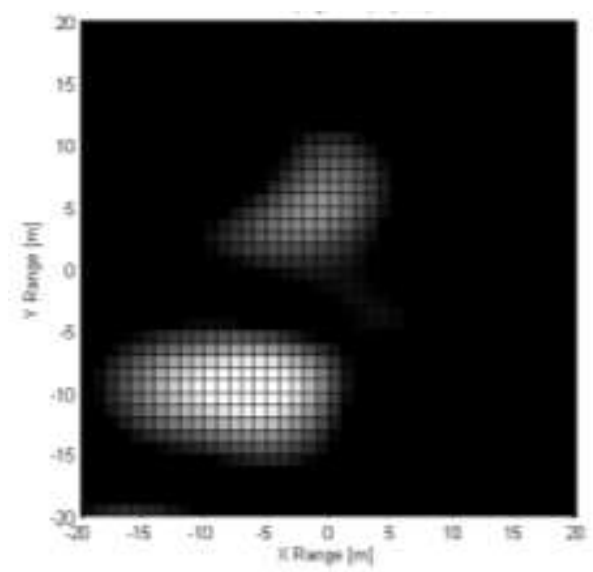

Figure 8: Reconstructed image using back-propagation.

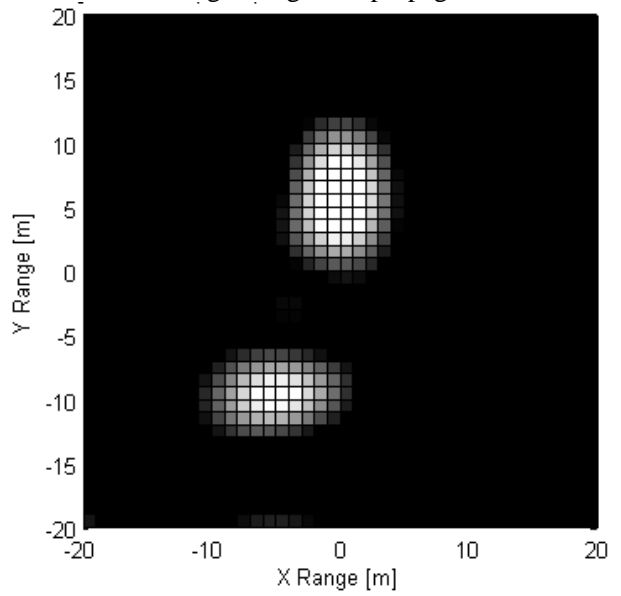

Figure 9: Reconstructed image using LM method by. $\beta$ has been empirically selected.

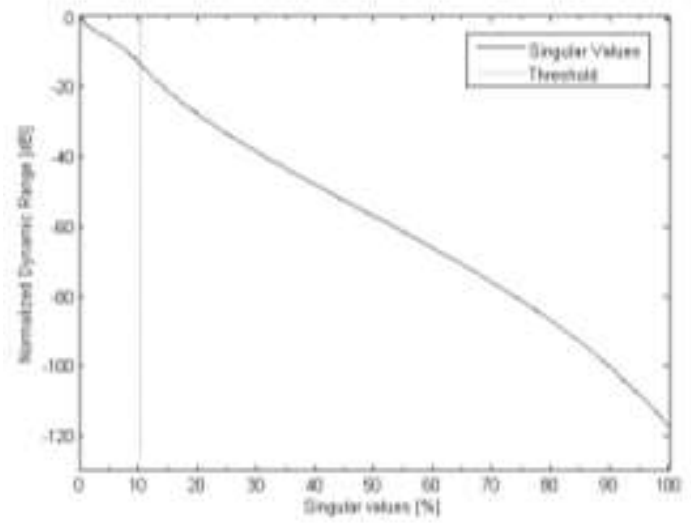

Figure 10: singular values behavior (in $\mathrm{dB}$ and normalized to the first singular value) of the $\mathbf{L}$ operator.

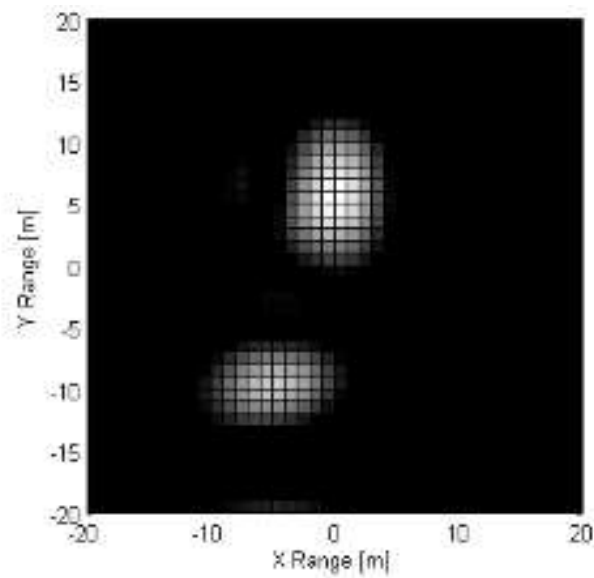

Figure 11: Reconstructed image using TSVD. The number of singular values used has been empirically selected.

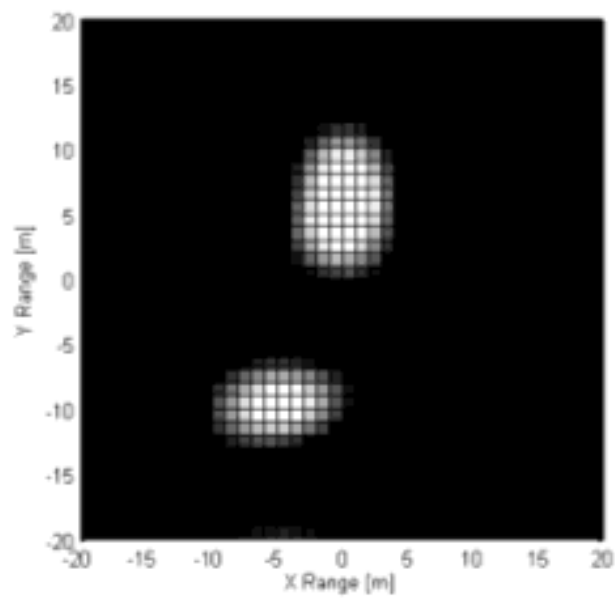

Figure 12: Reconstructed image using TSVD and scattered field data from the forward model in Section III.

\section{REFERENCES}

[1] I. Bishop, P. Styles, S. J. Emsley and N. S. Ferguson, "The Detection of Cavities Using the Microgravity Technique: Case Histories from Mining and Karstic Environments", Geological Survey, London, Engineering Geology Special Publications, Vol. 12, pp. 153-166, 1997.

[2] M. S. Zhdanov, Geophysical Inverse Theory and Regularization Problems, Methods in Geochemistry and Geophysics, Vol. 36, Elsevier, Amsterdam, 2002. 
[3] A. A. Kaufman, and R. O. Hansen, Principles of the Gravitational Method, Vol. 41, Elsevier, Amsterdam, Netherland, 2008.

[4] M. Hanke, and M. Brühl, "Recent Progress in Electrical Impedance Tomography”, Inverse Problems, Vol. 19, pp. 1-26, Nov. 2003.

[5] D. S. Holder, Electrical Impedance Tomography: Methods, History and Applications, Insitute of Physiscs, Series in Medical Physics and Biomedical Engineering, Ch. 10, London, UK, 2005.

[6] R. Miller et al, "Tunnel Detection Using Seismic Methods," Proc. AGU Meeting , Baltimore, MD, USA, NS21A-05, 2006.

[7] H. T. Andersen, and C. K. Skokan, "Seismic Tomography at the WIPP Site," SEG Proc. Fourth Tunnel Detection Symposium, pp. Golden CO, pp. 179-182, Apr. 26-29, 1993.

[8] W. H. Worthington, "An Introduction to Geophysical Tomography," First Break, pp. 20-26, Nov. 1984.

[9] V. V. Spichak, Electromagnetic Sounding of the Earth's Interior, Vol. 40, Elsevier, Amsterdam, Netherland, 2007.

[10] D. J. Daniels, Ground Penetrating Radars, Second Edition. Institution of Electrical Engineers, London, UK, 2004.

[11] R. C. Kemerait, "Tunnel Detection without the Use of Boreholes: What Are Our Capabilities and What Improvements Can Be Made?" SEG Proc. Fourth Tunnel Detection Symposium, Golden CO, pp. 497-515, Apr. 26-29, 1993.

[12] L. Stolarczyk, "Detection of Clandestine Tunnels, Bunkers and Weapon Caches with $1^{\text {st }}$ Interface Reflection Suppression Ground Penetrating Radar," Proc. 2008 Meeting for the Military Sensing Symposia (MSS), Specialty on Battlespace Acoustic and Seismic Sensing, Magnetic and Electric Field Sensors, Laurel, MD, USA, Aug. 19-21, 2008.

[13] T. J. Cui, W. C. Chew, A. A. Aydiner, D. L. Wright, and D. V. Smith, "Detection of Buried Targets Using a New Enhanced Very Early Time Electromagnetic (VETEM) Prototype System," IEEE Trans. Geosci. Remote Sens., Vol. GRS-29, No. 12, pp. 2702-2712, Dec. 2001.

[14] S. Shope, and R. J. Greenfield, "Electromagnetic Cross-Hole Tomography for Tunnel Detection," Third Technical Symposium on Tunnel Detection, Golden, CO, Jan. 12-15, 1988.

[15] R. J. Lytle, E. F Laine, D. L. Lager and D. J. Davis, "Using Cross Borehole Electromagnetic Probing to Locate High Contrast Anomalies," Geophysics. Vol. 44, pp. 1667-1676, 1979.

[16] I. C. Peden and J. Brew, "A laboratory scale model for the study of subsurface scattering in low-loss media with applications to ground penetrating radar," J. Appl. Geophys., vol. 33, pp. 109-118, 1995.

[17] J. R. Wait, Electromagnetic Probing in Geophysics, The Golem Press Boulder, CO, 1971.

[18] L. C. Bartel, D.H. Cress, and L.G. Stolarczyk, "Use of EM Gradiometer Concept for Detection of Underground Structures," Journal of Environmental and Engineering Geophysics, Vol. II, pp. 126-136, 1998.

[19] G. A. Ellis, and I. C. Peden, "An improved measurement facility for cross-borehole sensing," in Proc. 1992 IEEE Antennas and Propagation Int. Symp., Vol. 1, pp. 276-279, Jul. 1992.

[20] A. J. Witten and E. Long, "Geophysical imaging with arbitrary source illumination," IEEE Trans. Geosci. Remote Sensing, Vol. GRS-26, No. 4, pp. 409-419, Jul. 1988.

[21] G. A. Ellis and I. C. Peden, "Detection and location of buried dielectric objects using cross-borehole sensing," Progress in Electromagnetic Research Symp. (PIERS) Proc., p. 282, Jul. 1993.

[22] J. Schneider, J. Brew, and I. C. Peden, "Electromagnetic detection of buried dielectric targets," IEEE Trans. Geosci. Remote Sensing, Vol. 29, No. 4, pp. 555-562, Jul. 1991.

[23] G. A. Ellis, and I. C. Peden, "Cross-Borehole Sensing: Identification and Localization of Underground Tunnels in the Presence of a Horizontal Stratification," IEEE Trans. Geosci. Remote Sens., Vol GRS-35, No.3, pp. 756-761, May 1997.

[24] H. Zhou, M. Sato, "Subsurface Cavity Imaging by Crosshole Borehole Radar Measurements," IEEE Trans. Geosci. Remote Sens., Vol. GRS42, No. 2, pp. 335-341, Feb. 2004

[25] A. J. Devaney, "Geophysical Diffraction Tomography," IEEE Trans. Geosci. Remote Sens. Vol. GE-22, No. 3, pp. 3-13, Jan. 1984.

[26] A. J. Witten, and E. Long, "Shallow Applications of Geophysical Diffraction Tomography," IEEE Trans. Geosci. Remote Sens., Vol GE24, pp. 654-662, Sept. 1986
[27] A. J. Witten, J. E. Molyneux, and J. E. Nyquist, "Ground Penetrating Radar Tomography: Algorithms and Case Studies," IEEE Trans. on Geosci. Remote Sens., Vol. GRS-32, No. 2, pp. 461-467, Mar 1994.

[28] T. J. Cui, and W. C. Chew, "Diffraction Tomographic Algorithm for the Detection of Three-Dimensional Objects Buried in a Lossy Half-Space," IEEE Trans. Antennas Propag., Vol. AP-50, No. 1, pp. 42-49, Jan. 2002.

[29] T. B. Hansen, and P. M. Johansen, "Inversion Scheme for Ground Penetrating Radar that Takes Into Account the Planar Air-Soil Interface," IEEE Trans. Geosci. Remote Sens., Vol. GRS-38, No. 1, pp. 496-506, Jan. 2000

[30] P. Meincke, "Linear GPR Inversion for Lossy Soil and a Planar Air-Soil Interface," IEEE Trans. on Geosci. and Remote Sens., Vol. GRS-39, No. 12, pp. 2713-2721, Dec. 2001.

[31] G. A. Tsihrintzis, P. Meincke, and A. J. Devaney, "Buried Object Detection and Location Estimation from Electromagnetic Field Measurements," IEEE Trans. Antennas Propag., Vol. AP-47, No. 11, pp. 1742-1744, Nov. 1999.

[32] A. J. Devaney, "The limited-view problem in diffraction tomography" Inverse Problems, Vol. 5, No. 4, pp. 501-521, Aug. 1989

[33] M. C. Wicks, "RF Tomography with Application to Ground Penetrating Radars," IEEE Proc. $41^{\text {st }}$ Asilomar Conference ACSSC 2007, pp. 20172022, Nov. 4-7, 2007.

[34] G. Leone, and F. Soldovieri, "Analysis of the Distorted Born Approximation for Subsurface Reconstruction: Truncation and Uncertainities Effect," IEEE Trans. on Geosci. and Remote Sens., Vol. GRS-41, No. 1, pp. 66-74, Jan. 2003.

[35] R. Persico, R. Bernini, and F. Soldovieri, "The Role of the Measurement Configuration in Inverse Scattering from Buried Objects under the Born Approximation," IEEE Trans. Antennas and Propag., Vol. AP-53, No. 6, pp. 1875-1887, Jun 2005.

[36] H. J. Li, and F. L. Lin, "A Generalized Interpretation and Prediction in Microwave Imaging Involving Frequency and Angular Diversity," $J$. Electromagnetic Waves and Applications, Vol. 4, No. 5, pp. 415-430, 1990.

[37] K. M. Magde, and M. C. Wicks, "Waveforms in Virtual Tomographic Arrays," IEEE Proc. Waveform Diversity and Design Conference, pp. 83-87, 4-8 Jun. 2007.

[38] L. Lo Monte, D. Erricolo, and M. C. Wicks, "Propagation Model, Optimal Geometry and Receiver Design for RF Geotomography," IEEE Proc. RadarCon 2008, Rome, Italy, May 26-30, 2008.

[39] L. Lo Monte, A. M. Bagci, D. Erricolo, and R. Ansari, "Spatial Resolution in Tomographic Imaging with Diffracted Fields," Proc. XXIX General Assembly of the International Union of Radio Science (URSI), Chicago, IL, USA, Aug. 7-16, 2008.

[40] L. Lo Monte, and D. Erricolo, "Distributed RF Tomography for Void Detection," Proc. 2008 Meeting for the Military Sensing Symposia (MSS), Specialty on Battlespace Acoustic and Seismic Sensing, Magnetic and Electric Field Sensors, Laurel, MD, USA, Aug. 19-21, 2008

[41] J. Norgard, M. C. Wicks, and A. Drozd, "Distributed/Embedded SubSurface Sensors for Imaging Buried Objects with Reduced Mutual Coupling and Suppressed Electromagnetic Emissions," Proc. International Conference on Electromagnetics in Advanced Applications (ICEAA), pp. 427-430, Turin, Sept. 17-21, 2007.

[42] L. Lo Monte, and D. Erricolo, "Receiving Antenna Design for Ground Penetrating Tomographic Imaging”, Proc. IEEE AP-S International Symposium, San Diego, CA, USA, Jul. 5-12, pp. 1-4, 2008.

[43] F. Soldovieri, J. Hugenschmidt, R. Persico, and G. Leone, "A Linear Inverse Scattering Algorithm for Realistic GPR Applications," Near Surface Geophysics, Vol. 5, No. 1, pp. 29-42, Feb. 2007.

[44] W. C. Chew, Waves and Fields in Inhomogeneous Media, IEEE Press, Piscataway NJ, 1995.

[45] C. T. Tai, Dyadic Green Functions in Electromagnetic Theory, Second Edition, IEEE Press, Piscataway, NJ, 1993

[46] T. B. Hansen, and P. M. Johansen, "Inversion Scheme for Ground Penetrating Radar that Takes into Account the Planar Air-Soil Interface," IEEE Trans. on Geosci. Remote Sens., Vol. GRS-38, No. 1, pp. 496-506, Jan. 2000.

[47] T. K. Moon, and W. C. Stirling, Mathematical Methods and Algorithms for Signal Processing, Ch. 4, Prentice Hall, Upper Saddle River, NJ, 2000 
[48] M. Cheney, "A mathematical tutorial on Synthetic Aperture Radar", SIAM Review, Vol. 43, No. 2, pp. 301-312, 2001.

[49] T. J. Cui, W. C. Chew, X. X. Yin, and W. Hong, "Study of Resolution and Super Resolution in Electromagnetic Imaging for Half-Space Problems," IEEE Trans. Antennas Propag. Vol. AP-52, No. 6, pp. 1398-1411, Jun. 2004.

[50] S. X. Pan, and A. C. Kak, "A Computational Study of Reconstruction Algorithms for Diffraction Tomography: Interpolation vs. Filtered Backpropagation," IEEE Trans. on Acoust. Speech, Signal Process., Vol. ASSP-31, pp. 1262-1275, Oct. 1983.

[51] F. A. Marvasti, Nonuniform Sampling, Theory and Practice, Kluwer Academic/Plenum Publisher, New York, NY, 2001.

[52] R. W. P. King, and G. S. Smith, Antennas in Matter, Ch. 11, MIT Press, Cambridge MA, 1981.

[53] R. W. P. King, B. H. Sandler, and L. C. Shen, "A Comprehensive Study of Subsurface Propagation from Horizontal Electric Dipoles," IEEE Trans. Geosci. Remote Sens., Vol. GRS-18, No. 3, pp. 225-233, Jul. 1980.

[54] R. W. P. King, M. Owens, and T. T.Wu, Lateral Electromagnetic Waves. Springer-Verlag, New York, NY, 1992.

[55] P. Van Etten, and R. D. Brown, "System and Method for Earth Probing with Deep Subsurface Penetration Using Low-Frequency Electromagnetic Signals," US Patent N. 5,357,253, Oct 1994.

[56] J. L. Volakis, Antenna Engineering Handbook, Fourth Edition, McGraw Hill, New York, NY, 2007.

[57] B. Simon, Quantum Mechanics for Hamiltonians Defined as Quadratic Forms, Princeton University Press, New Jersey, 1971.

[58] D. A. Hill, "Gradiometer antennas for tunnel detection," Nat. Inst. Stand. Tech. (U.S.) NISTIR, 3990, June 1992.

[59] R. C. Pettengill, H. T. Garland, and J. D. Meindl, "Receiving Antenna Design for Miniature Receivers", IEEE Trans. Antennas Prop., Vol. AP-25, No. 4, pp. 528-530, Jul. 1977.

[60] A. Pralat, and R. Zdunek, "Electromagnetic Geotomography-Selection of Measuring Frequency," IEEE Sensor J., Vol. SEN-5, No. 2, pp. 242250, Apr. 2005.

[61] G. R. Olhoeft, "Electrical Properties of Rocks" [book auth.] Y. S. Touloukian, W. R. Judd and R. F. Roy, Physical Properties of Rocks and Minerals. Hemisphere Publishing Corporation, pp. 257-329, New York, NY 1989.

[62] E. I. Parkhomenko, Electrical Properties of Rocks, Plenum Press, New York, NY, 1967.

[63] ITU-R, Recommendation No. ITU-R-P372-9.

[64] G. A. Tsihrintzis, and A. J. Devaney "High Order (Nonlinear) Diffraction Tomography: Inversion of the Rytov Series," IEEE Trans. Inf. Theory. Vol. IT-46, No. 5, pp. 1748-1761, Aug. 2000.

[65] C. A. Balanis, Advanced Engineering Electromagnetics, Wiley Interscience, New York, NY, 2005.

[66] M. Bertero, and P. Boccacci, Introduction to Inverse Problems in Imaging, Institute of Physics Ltd, London, UK, 2002.

[67] T. Varslot, B. Yazici, M. Cheney, "Wide-Band Pulse-Echo Imaging with Distributed Apertures in Multi-Path Environments", Inverse Problems, Vol. 24, No. 4, pp. 1-28, Aug. 2008.

[68] A. C. Kak, M. Slaney, Principles of Computerized Tomographic Imaging, SIAM, Philadelphia, PA, 2001.

[69] P. C. Hansen, Rank Deficient and Discrete Ill-Posed Problems, SIAM, Philadelphia, PA, 1998.

[70] A. Giannopoulos, GPRMAX Simulator, www.gprmax.org

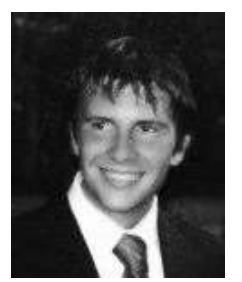

Lorenzo Lo Monte (S'05) received his B.Sc. and M.Sc. (both summa cum laude) in Telecomunnications engineering from University of Rome "Tor Vergata", Rome, Italy in 2003 and 2005, respectively. He is currently pursuing his Ph.D. degree in Electrical Engineering at the University of Illinois at Chicago (UIC), Chicago, IL, USA.

In 2005, he worked at the Rheinmetall AG facility in Rome, Italy, for the synthesis of phased arrays for antiaircraft systems. In 2007 he worked as a contractor for the Air Force Research Laboratory / Sensor Directorate on RF sensing and electromagnetic imaging. From 2007 to 2008, he worked at PCTEL Inc. as antenna engineer, developing a new family of WiMax BTS sector panels. Since March 2008 he is working at General Dynamics Information Technology in Dayton, $\mathrm{OH}$ as a contractor for Air Force Research Laboratory / RYRT branch at Wright Patterson Air Force Base, Dayton, OH. On February 2009, he joined the Rennselaer Polytechnic Institute, Troy, NY as a visiting scholar, performing active research in inverse problems in radar imaging. His main research interests include radar imaging, electromagnetic imaging, subsurface sensing and inverse scattering.

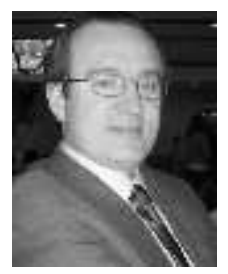

Danilo Erricolo (S'97-M'99-SM'03) received the Laurea degree of Doctor in electronics engineering (summa cum laude) from the Politecnico di Milano, Milan, Italy, in 1993 and the Ph.D. degree in electrical engineering and computer science from the University of Illinois at Chicago (UIC), in 1998.

$\mathrm{He}$ is an Associate Professor in the Electrical and Computer Engineering Department, UIC, where he also is Associate Director of the Andrew Electromagnetics Laboratory. His research interests are primarily in the areas of wireless communications, electromagnetic scattering, and electromagnetic compatibility. His research activity is supported by the National Science Foundation and the Department of Defense. He has authored or coauthored more than 100 publications in refereed journals and international conferences.

Dr. Erricolo is a Member of Eta Kappa Nu and was elected a Full Member of the U.S. National Committee of the International Union of Radio Science (USNC-URSI) Commission B and E. He serves as Vice-Chair for the USNCURSI Commission E. He was twice awarded both the Andrew Foundation Fellowship and the Beltrami Foundation Fellowship. He is an Associate Editor for the IEEE ANTENNAS AND WiRELess PROPAGATION LETTERS. He also has served as Vice Chair of the Local Organizing Committee of the XXIX URSI General Assembly and has been appointed as General Chair of the 2012 IEEE Antennas and Propagation International Symposium/USNC National Radio Science Meeting in Chicago, IL.

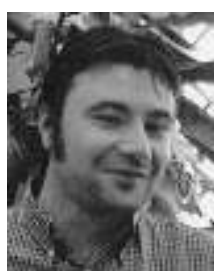

Francesco Soldovieri received the Laurea degree in electronic engineering from the University of Salerno, Salerno, Italy, in 1992 and the Ph.D. degree in electronic engineering from the University of Naples "Federico II," Naples, Italy, in 1996.

In 1993, he joined the Electromagnetic Research Group of the University of Naples, and in 1998/1999, he held a Postdoctoral Fellowship at the same University. In 1999-2000, he collaborated with the research group on Applied Electromagnetism of the Second University of Naples. Since 2001, he has been a Researcher with the Institute for the Electromagnetic Sensing of the Environment of the Italian National Research Council, Naples. His current main scientific interests include electromagnetic diagnostics, inverse scattering, ground-penetrating-radar applications, and antenna diagnostics.

Dr. Soldovieri was awarded the 1999 Honorable Mention for the H.A. Wheeler Applications Prize Paper Award of the IEEE Antennas and Propagation Society.

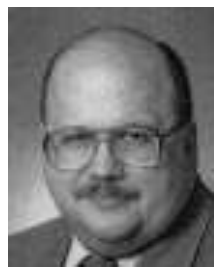

Michael C. Wicks (S'81-M'89-SM'90-F'98) received the undergraduate degrees from Mohawk Valley Community College and Rensselaer Polytechnic Institute, Troy, NY, and the graduate degrees from Syracuse University, Syracuse, NY, all in electrical engineering.

$\mathrm{He}$ is a Principal Research Engineer in the U.S. Air Force Research Laboratory in the Sensors Directorate, Radar Signal Processing Branch. He has authored over 125 papers, reports, and patents. His interests include adaptive radar signal processing, wide-band radar technology, ground penetrating radar, radar clutter characterization, and knowledge-based applications to advanced signal processing algorithms, detection, and estimation theory and applied statistics.

Dr. Wicks serves on the board of the SUNY Institute of Technology Foundation, the Mohawk Valley Community College Engineering Science Advisory Council, and the IEEE Aerospace and Electronic Systems Board of Governors and is chairman of the IEEE Radar Systems Panel. 